\title{
The Impact of COVID-19 on Acute and Elective Corneal Surgery at Moorfields Eye Hospital London
}

\author{
Nizar Din' \\ Maria Phylactou' \\ Julia Fajardo-Sanchez' \\ Martin Watson' \\ Sajjad Ahmad ${ }^{1,2}$ \\ 'Cornea and External Eye Disease \\ Service, Moorfields Eye Hospital, \\ London, UK; ${ }^{2}$ Institute of \\ Ophthalmology, University College \\ London, London, UK
}

Purpose: Moorfields Eye Hospital sits as a major tertiary centre for ophthalmic care in the United Kingdom and became a central hub to provide safe and effective ophthalmic care across London and surrounding regions during the COVID-19 pandemic. We explore the impact on both the acute and elective corneal services during the first wave of this pandemic. Methods: A retrospective review of the proportion of corneal transplants and anterior segment trauma repairs was performed during the period of March 23rd to July 1st 2020 compared with an identical period in 2019. Data were acquired from our in-house electronic patient records.

Results: A 92\% reduction in corneal elective work was observed during the lockdown period compared with an identical period in 2019, with only 10 elective cases in total being performed. In addition, 91 corneal cross-linking and 76 therapeutic lasers were cancelled. There were 15 cases of primary repair for anterior segment trauma compared with 6 cases pre-COVID-19. A similar scenario occurs with removal of foreign body (4 cases during COVID-19 period versus no cases during pre-COVID-19 era) and with traumatic lens aspirations (6 cases during COVID-19 compared with 2 pre-COVID-19). Interestingly, a statistical difference $(\mathrm{p}=0.03)$ was found in the time interval from presentation of symptoms to emergency corneal surgery. During the COVID-19 period, a delay of 1.5 days \pm 2.29 (range 0-10 days) occurred compared with 0.8 days \pm 1.54 (range 0-6 days) pre-COVID-19. Conclusion: Stringent risk stratification reduced elective corneal surgery capacity during the lockdown thereby preserving social distancing requirements. However, an apparent increase in emergency corneal surgery seen is likely attributed to centralisation of ophthalmic services during the pandemic crisis, alongside increased domestic injuries. Despite the challenges posed, successful delivery of corneal surgery occurred whilst helping to identify lessons in preparations for future pandemics and current inefficiencies in healthcare delivery.

Keywords: COVID-19, corneal surgery, perforations, trauma
Correspondence: Nizar Din

Moorfields Eye Hospital, 162 City Road,

London, ECIV 2PD, UK

Email nizar.din@nhs.net

\section{Introduction}

Moorfields Eye Hospital (MEH) NHS Foundation Trust is responsible for over 20\% of all corneal graft surgery nationally (A Rahman, Eye Bank Manager, Moorfields Lion Eye Bank, email communication, July, 2020). An NHS Trust is an organisational unit within the National Health Service in England and Wales, generally serving either a geographical area or a specialised function. $\mathrm{MEH}$ is one of these Trusts which provides specialised tertiary level ophthalmic care to the greater London area. Foreseeing that other ophthalmic units across London would end up 
shutting or ophthalmic staff being redeployed, MEH became a central hub to provide safe and effective ophthalmic care during the COVID-19 pandemic across London and surrounding regions. As a consequence, the Trust quickly restructured its services to provide only emergency and urgent sight threatening care from March 23rd 2020.

MEH consists of 26 outreach sites across London, and it was decided to centralise all services to its main centre at City Road, citing host hospitals at these outreach sites becoming overwhelmed with COVID-19 cases, the need for additional bed space and the reduction of elective ophthalmic care. Furthermore, it was forecast that the Accident and Emergency service at Moorfields would absorb increasing number of emergencies, with a consequent knock on effect on the cornea and external diseases services.

A London-wide strategy positioned MEH as a central site to provide ophthalmic services, so that other peripheral eye hospitals can allow their staff to be redeployed to the frontline combating COVID-19. Hence, it was forecasted that the general Accident and Emergency service at Moorfields would absorb increasing number of emergencies, which would mean increased referrals and triaging to the cornea and external diseases services.

We explore the impact on both the acute and elective corneal services during this first wave of this pandemic.

\section{Methods}

A retrospective review of the proportion of corneal transplants and anterior segment trauma repairs performed during the period of March 23rd to July 1st 2020 compared with the identical period in 2019. Data were acquired from our in-house electronic patient records. Further information regarding corneal tissue procurement was obtained through the Moorfields Lions eye bank. Inclusion criteria for this study were all new patients who were referred from the emergency ophthalmic department at MEH to the on-call corneal service for surgical intervention. Furthermore, any existing patients known to the department who required emergent intervention were also included. The second parallel arm included any elective patients who were scheduled for surgery during the period specified. There was no exclusion criteria as we aimed to comprehensively evaluate the impact on the corneal surgical service on both elective and emergency cases. Statistical analysis was performed using the Statistical Package for the Social Sciences (SPSS) (IBM, U.S). The study was approved by the Clinical Audit and Effectiveness Committee at Moorfields Eye Hospital NHS Foundation Trust (Audit Number: 647) and was adherent to the tenets of the Declaration of Helsinki. Patients' characteristics and surgical data were reviewed through the Electronic medical records (Open Eyes v1.18, www.openeyes.org.uk). No prior patient consent was required as all data were confidential by removing identifiable matrices.

\section{Results}

As per Public Health England (PHE) guidance and RCOphth guidance, ${ }^{1,2}$ elective care was cancelled and a total of 128 corneal graft cases were risk stratified according to risk of sight loss. This risk stratification profile was categorised into the following four groups:

- Group 1 - Urgent cases where irreversible loss of vision is likely within 3 months; emergency work; urgent oncology.

- Group 2 - Urgent cases where irreversible loss of vision likely within 6 months

- Group 3 - Restoring vision in only eye or allows patient to return to work.

- Group 4 - Improving visual acuity, quality of life, independence.

From March 23rd to 1st July 2020, only 10 elective cases were performed - 5 Descemet Membrane Endothelial Keratoplasty (DMEK), 3 Descemet Stripping Automated Endothelial Keratoplasty (DSAEK), 1 Penetrating Keratoplasty (PKP) and 1 limbal dermoid for amblyopia prevention. Compared with an identical period in 2019 (pre-COVID-19) where a total of 163 graft surgeries were performed, this was a drop of elective work by $92 \%$ (see Figure 1). Many of these elective cases during COVID-19, were performed towards the tail end of June coinciding with the Trust's recovery phase. Additionally, during this period, 91 corneal cross-linking (CXL) and 76 therapeutic laser procedures were also cancelled citing risk of aerosolization from plume smoke during laser intervention. A total of 21 from 24 donor corneal tissues were used during the lockdown period while 181 corneal tissues were utilised during the pre-COVID period.

During the lockdown period of March 23rd to 1st July 2020, 38 emergency corneal procedures were performed, which was identical to the 2019 period (Figure 2). However, it is interesting to note that during 


\section{Elective Surgeries performed during Pre-Covid and Covid periods divided by Months}

25

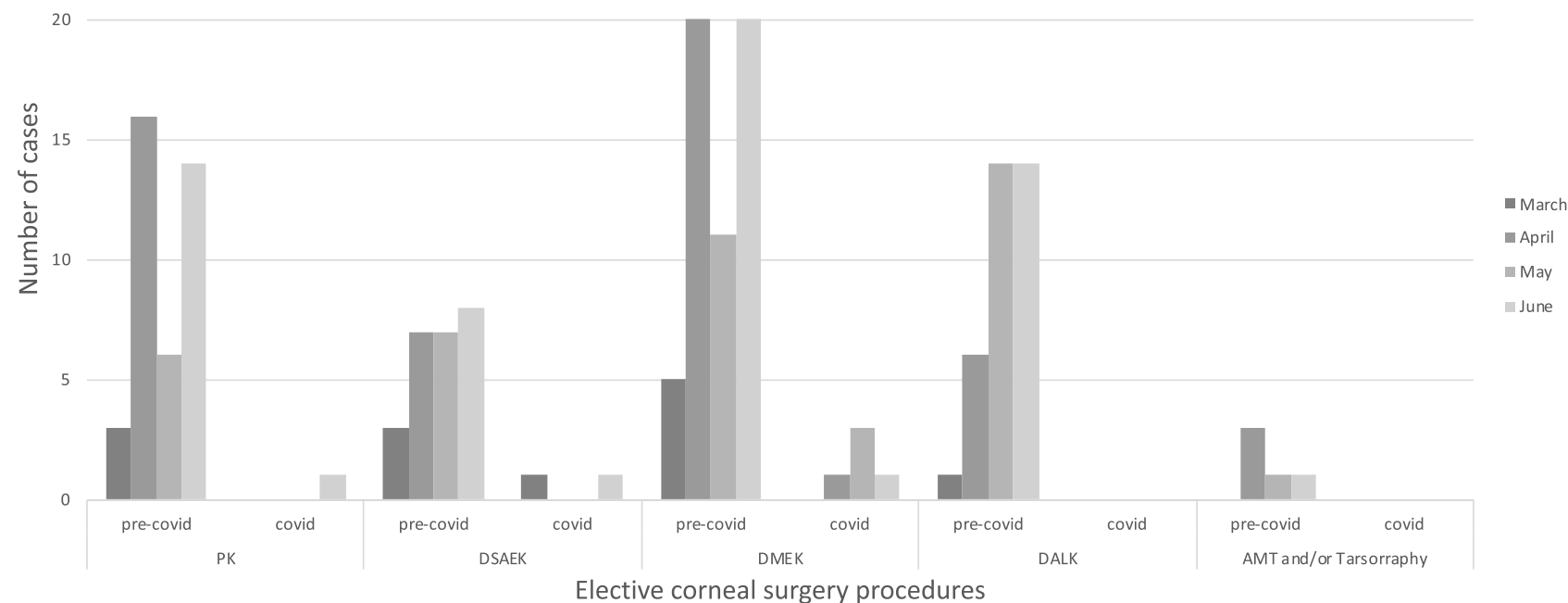

Figure I Elective corneal surgeries performed during pre-COVID-19 period and COVID period.

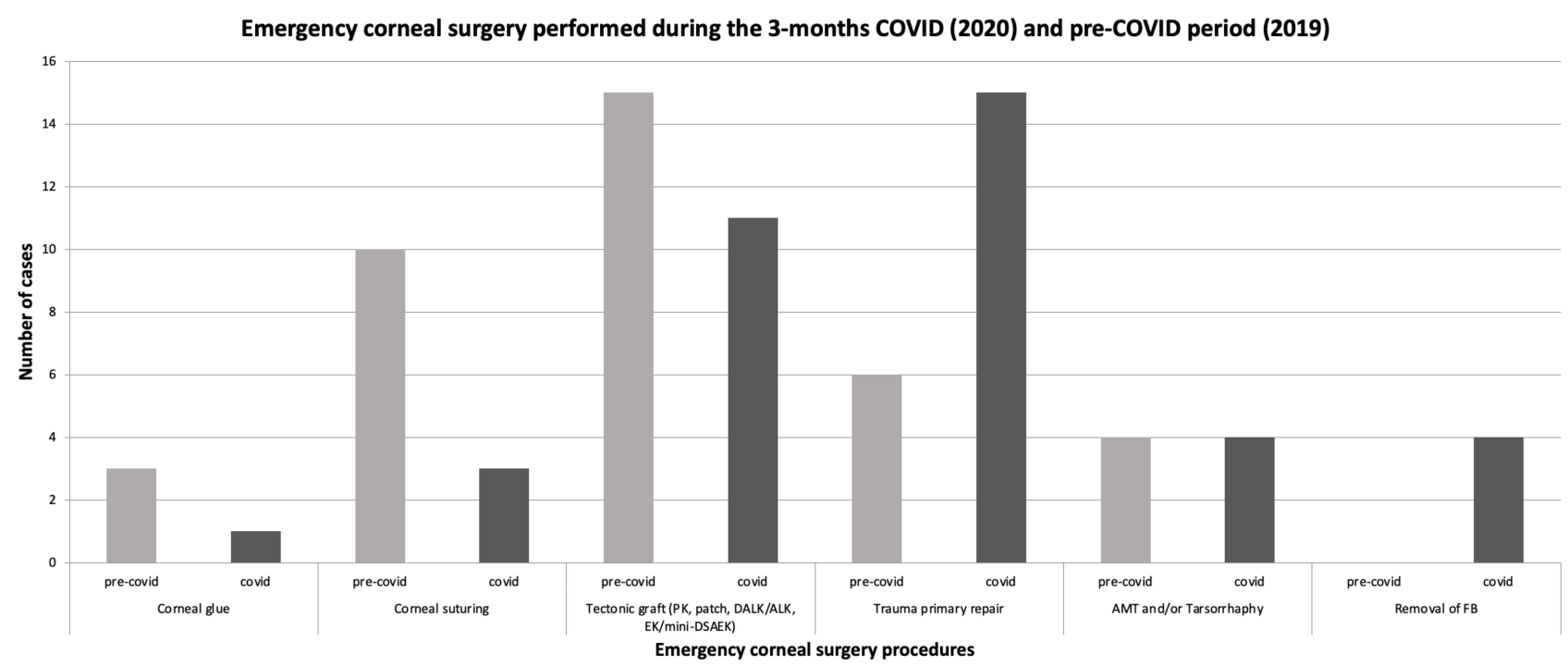

Figure 2 Emergency corneal surgeries performed during pre-COVID-19 period and COVID period.

COVID-19 period primary repair surgery for anterior trauma was performed for 15 cases in comparison with the 6 cases attended for the same type of surgery during pre-COVID-19 times. A similar scenario occurs with removal of foreign body (FB) (4 cases during COVID19 period versus no cases during pre-COVID-19 times) and with traumatic lens aspirations (6 cases during COVID-19 period versus only 2 in pre-COVID-19). When we look at indications for emergency surgery, "Sharp object trauma" was classified as either penetrating injury with or without FB removal in addition to cornea or sclera-corneal lacerations. Considering this, 13 cases presenting sharp object trauma were attended during COVID19 period while only 1 case was seen in pre-COVID-19 frame time (Table 1). Finally, 11 cases of tectonic grafts were performed during COVID times versus 15 during pre-COVID-19 period. This difference is correspondent to the number of cases of corneal melts with perforation seen in both periods (13 in COVID-19 and 18 in preCOVID-19). 
Table I Number of Cases per Indication for Emergency Surgery in Pre-COVID and COVID Periods

\begin{tabular}{|c|c|c|}
\hline Indications for Emergency Surgery & Pre-COVID & COVID \\
\hline \multicolumn{3}{|l|}{ Blunt Trauma } \\
\hline Globe rupture & 3 & 3 \\
\hline Graft wound dehiscence & 8 & 4 \\
\hline Traumatic & 7 & 4 \\
\hline Non-traumatic & 1 & 0 \\
\hline \multicolumn{3}{|l|}{ Sharp Object Trauma } \\
\hline Penetrating Injury & 0 & 4 \\
\hline With IOFB & 0 & 2 \\
\hline Without IOFB & 0 & 2 \\
\hline Laceration (Corneal, Scleral, & 1 & 9 \\
\hline Corneoscleral) & & \\
\hline Full thickness & I & 8 \\
\hline Partial thickness & 0 & 1 \\
\hline \multicolumn{3}{|l|}{ Other } \\
\hline $\begin{array}{l}\text { Corneal melt (infection, inflamatory, } \\
\text { LSCD, neurotrophic, other) }\end{array}$ & 19 & 13 \\
\hline With perforation & 18 & 13 \\
\hline Without perforation & 1 & 0 \\
\hline PED & 2 & 3 \\
\hline $\begin{array}{l}\text { Post-Graft related complications } \\
\text { (brooken suture, loose suture, GHJ } \\
\text { leak) }\end{array}$ & 5 & 1 \\
\hline \multirow[t]{2}{*}{ Conjunctival FB } & 0 & 1 \\
\hline & 38 & 38 \\
\hline
\end{tabular}

Each elective corneal case had a single donor tissue assigned. Each day the supply is stocked with two full thickness cornea donors and two sheets of amnion membrane for emergency cornea cases. In the unlikely event of multiple emergencies surpassing the designated quota, then extra tissues can be ordered within a 48-hour window.

Interestingly, a statistical difference $(\mathrm{p}=0.03)$ was found in the time interval from presentation of symptoms to emergency surgery under the corneal team. During the COVID19 period, a delay of 1.5 days \pm 2.29 (range $0-10$ days) compared with 0.8 days \pm 1.54 (range $0-6$ days) preCOVID-19. A further analysis of distances emergency patients travelled during the COVID-19 period revealed that $37 \%$ of the patients came from outside the Greater London postcode, averaging 24.5 miles. This is in contrast to $21 \%$ of patients travelling from outside the Greater London postcode, averaging 20 miles in 2019. Our results show that the distances travelled during the COVID-19 (2020) and pre-COVID-19 (2019) years were not statistically significant $(\mathrm{P}>0.05)$, although there was a trend towards patients travelling further afield during the COVID-19 period which is consistent with the pooling of services centrally.

\section{Discussion}

There is a paucity of data on the impact of corneal graft surgery and the tissue bank during the "first wave" of the COVID-19 pandemic. The UK government's lockdown period included strict quarantine measures, social restriction measures and a stay-at-home order. Only essential services were allowed to open. As a result of these national guidelines, $\mathrm{MEH}$ de-escalated all services to an emergency only practice or catering for high-risk elective patients.

At MEH there were less tectonic grafts performed during the COVID-19 period compared with the equivalent in 2019, with fewer corneal melts with perforation being seen. As the pandemic took hold, the Lions Moorfields Eye Bank made a decision to always have on hold 2 emergency tissues and to regularly monitor corneal tissue usage weekly. In total, 24 donor corneal tissues were procured with 21 corneal tissues being utilised during the lockdown period. This is in comparison to 181 corneal tissues utilised in the same pre-COVID period. All donor tissues are currently being tested for COVID-19. This includes nose, throat and endotracheal donor swabs for COVID-19 Polymerase Chain Reaction (PCR) and blood serological testing. In Hong Kong, Tang et al reported the suspension of active solicitation of eye tissues; the inclusion of rapid tests for COVID-19 virus in the regular screening of donors; and the exclusion of donors if they presented with any respiratory symptoms or if they had been exposed to areas where outbreaks were reported. ${ }^{3}$ In Italy, Busin et al have also described an imbalance between supply and demand of corneal tissue during the pandemic. ${ }^{4}$ They reported that the Veneto Eye Bank saw a reduction of $95 \%$ in demand, compared with our experience of $87 \%$. There was no difference in the usability of tissues, although there were anecdotal trends of younger donors being provided. At the beginning of the lockdown, there were no issues with the supplies of tissues. However, as the lockdown period progressed, harvesting of donor tissues reduced but this did not affect delivery of tissues to MEH. No cases needed cancellation due to tissue shortages. The COVID-19 pandemic will have macro-economic and potentially long-lasting impact on corneal transplantations worldwide. ${ }^{7}$

The policies outlined above were dictated by a number of national and international recommendations. The American Academy of Ophthalmology (AAO), the 
world's largest association of eye physicians and surgeons, recommended on 18 March 2020 that all ophthalmologists immediately stop any treatment other than urgent or emergent care, thus including both office-based care and surgical care. ${ }^{8}$ With this statement, there remained a grey area in the definition of "urgency". This was left to the doctor's discretion, who had to take into account the social and medical circumstances of the individual patient. ${ }^{9}$ On 16 th April 2020, the US federal government recommended resumption of some facilities on the proviso that additional protection be sought including mouth protection, N95 masks, eye shields and slit-lamp breath shields when caring for COVID-19 patients. Furthermore, social distancing, frequent disinfection of rooms, and the wearing of face masks by both patients and caregivers were mandatory. ${ }^{9}$ All these policies implied a reduction in the volumes of patients to be visited and treated surgically compared to the pre-pandemic period. Meanwhile, in the UK, the Royal College of Ophthalmologists recommended a reduction in the length of surgical procedures by indicating only the most experienced and senior surgeons as operators, and excluding younger surgeons. This policy inadvertently placed senior clinicians who by default are at higher risk of developing COVID-19 to further dangers whilst simultaneously having repercussions on training programmes for the juniors. ${ }^{10}$ Amongst all of this is the ethical dilemma of balancing the risks of infection to both patients and doctors, against the risk of potential lawsuits of inaction or action. Many procedures in ophthalmology produce bioaerosols especially if the patient is intubated. This has created an additional layer of difficult clinical decision making as surgeons have to decide whether to operate or not, and re-addresses the risk/benefit assessment profile. With lack of consistent clinical protocols and supportive legal status, surgeons are increasingly exposed to lawsuits during this pandemic. Whilst the elimination of COVID-19 to zero is not possible, prudent adjustment and risk management profile is necessary. ${ }^{11,12}$

In the paper by Napoli et al, they describe the relevance of the ACE2 receptors and TMPRSS2 proteins on ocular transmission of the SARS-CoV2 virus. ${ }^{1,13}$ Whilst the risk of coronavirus transmission remains low through tears, there is still a risk that the SARS-CoV2 virus may survive for a long time or replicate in the conjunctiva, even after conjunctivitis symptoms are finished. Hence, eye care providers are encouraged to wear eye protection (protective goggles or in association with a face shield). ${ }^{14-16}$ These prevent risk of contaminated droplets and bioaerosols being spread by patients. MEH continued to follow public health guidelines including the World Health Organisation (WHO) who advocated limiting patient proximity in hospital waiting rooms. ${ }^{17}$ Furthermore, high risk immunocompromised patients were also risk stratified to stay at home. As a result, the foot flow dramatically decreased and only acute patients or elective impending corneal emergency patients presented to $\mathrm{MEH} .{ }^{18}$

The comparative increase in trauma-related corneal injuries during the COVID-19 period is likely borne out of increased time spent indoors leading in home improvement project-related injuries. There are conflicting data in the literature related to trauma during COVID-19, with Du et al reporting eye trauma surgery as rare, ${ }^{6}$ whilst Tang et al report that in a tertiary hospital in Hong Kong, 15.8\% of all cases were related to globe rupture. ${ }^{4}$ There is a paucity of reports on the impact of corneal surgery during COVID-19. Only Busin et al describe the Italian experience of corneal surgery, reporting 4 surgical cases in April. ${ }^{5}$ The observed fewer cases of infective keratitis induced corneal perforations may be attributable to a reduced use of contact lens wear, as more people work from home and have diminished social activities.

Of interest is the delay in presentation during the COVID-19 period. Whilst statistically significant, it demonstrates a worrying trend of patients neglecting their ocular symptoms. People were afraid to travel, especially in the first lockdown. The general consensus was life outweighed sight loss, with people delaying travelling to the emergency A\&E eye department because of the coronavirus travel restrictions and fear of infection. In the early days of lockdown, telemedicine was not widely available and it took a few weeks for this to be fully established. This corroborates with other sub-specialities, such as vitreoretinal, which have seen a $62 \%$ drop of retinal detachments rates. ${ }^{7}$ Possible explanations for this include the fear of presenting to a high risk environment such as a hospital, the closure of optometry practices, perceived lack of GP access or poor patient education. ${ }^{7}$ Despite a concerted media campaign by NHS England and Government officials, such delays in presentation will have a significant long-term impact on ocular health.

In conclusion, we suggest a number of key changes to be implemented for a successful transition to normal services whilst preparing for a subsequent second or third waves. Firstly, increased collaborative work with community optometrists and family doctors will be necessary to counterbalance the increased demand for corneal services as the economy and optometry practices reopens. During the 
lockdown period, centralisation of ophthalmic services to Moorfields Eye Hospital risked overburdening the emergency corneal services with the hospital becoming a potential incubating site for COVID-19 to spread. As a result, spreading the workload in the community with optometry colleagues reduces the volume of patients entering the hospital whilst complying with social distancing rules. Furthermore, it reduced the travel time for patients and hence the risk of contracting the virus. This partnership reserves only the most severe corneal complications to be dealt with in the hospital. Secondly, whilst elective corneal surgery is largely reversible, any recovery planning must continue to buffer against emergency work, and take into account the restriction of patient numbers in hospital due to social distancing. This will require close liaison with eye banks to meet the expected tissue demand in light of the significantly reduced organ donation during this period. Thirdly, any elective corneal surgery will require further re-stratification taking into account patients' age, co-morbidities, impact on quality of life and ocular health. This process of risk stratification is made more efficient due to readily available data from electronic medical records. Finally, in order to free up resources and clinic space for more urgent cases and emergency work, we will need to further utilise our newly established tele/video virtual clinics for low-risk patients. As the pandemic unfolded, new strategies were employed to deliver healthcare. Near the latter end of the lockdown period, virtual consults and telemedicine were being employed across the emergency ophthalmic services. Whilst the COVID-19 pandemic has had a significant impact on clinical and surgical provision, it is also an opportunity to address inefficiencies in our practices whilst coming up with new innovative concepts and collaborative links.

\section{Funding}

The publication costs of this article have been enabled by a grant from Moorfields Eye Charity [GR001331].

\section{Disclosure}

None of the authors have any proprietary interests or conflicts of interest related to this paper.

\section{References}

1. COVID-19 daily deaths. NHS England. Coronavirus (COVID-19). 2020. Available from: https://www.england.nhs.uk/statistics/statisticalwork-areas/covid-19-daily-deaths/. Accessed July 20, 2020.
2. COVID-19 clinical guidance for ophthalmologists. Royal College of Ophthalmologist; 2020. Available from: https://www.rcophth.ac.uk/ 2020/05/covid-19-update-and-resources-for-ophthalmologists/.

Accessed July 20, 2020.

3. Tang EWH, Wong DHT, Chan YYY, Li KKW. Emergency ophthalmic surgeries during COVID-19 - a Hong Kong perspective. Graefes Arch Clin Exp Ophthalmol. 2020;258(12):2867-2868. doi:10.1007/ s00417-020-04768-8

4. Busin M, Yu AC, Ponzin D. Coping with COVID-19: an Italian perspective on corneal surgery and eye banking in the time of a pandemic and beyond. Ophthalmology. 2020;127(9):e68-e69. doi:10.1016/j.ophtha.2020.04.031

5. Du H, Zhang M, Zhang H, Sun X. Practical experience on emergency ophthalmic surgery during the prevalence of COVID-19. Graefes Arch Clin Exp Ophthalmol. 2020;258(8):1831-1833. doi:10.1007/ s00417-020-04692-x

6. Wickham L, Hay G, Hamilton R, et al. The impact of COVID policies on acute ophthalmology services-experiences from Moorfields Eye Hospital NHS Foundation Trust. Eye (Lond). 2020;34(7):1189-1192. doi:10.1038/s41433-020-0957-2

7. Ang M, Moriyama A, Colby K, et al. Corneal transplantation in the aftermath of the COVID-19 pandemic: an international perspective. Br J Ophthalmol. 2020;104(11):1477-1481. doi:10.1136/bjophthalmol-2020-317013

8. American Academy of Ophthalmology. Recommendations for urgent and nonurgent patient care. San Francisco, CA, USA: American Academy of Ophthalmology; 2020. Available from: https://www. aao.org/headline/new-recommendations-urgent-nonurgent-patientcare. Accessed May 1, 2020.

9. Napoli PE, Nioi M, d'Aloja E, Fossarello M. Safety recommendations and medical liability in ocular surgery during the COVID-19 pandemic: an unsolved dilemma. J Clin Med. 2020;9(5):1403. doi: $10.3390 / \mathrm{jcm} 9051403$

10. The Royal College of Ophthalmology. COVID-19 clinical guidance for ophthalmologists. London, UK: The Royal College of Ophthalmology; 2020. Available from: https://www.rcophth.ac.uk/ 2020/04/covid-19-update-and-resources-for-ophthalmologists. Accessed May 1, 2020.

11. Adams JG, Walls RM. Supporting the health care workforce during the COVID-19 global epidemic. JAMA. 2020;323(15):1439-1440. doi:10.1001/jama.2020.3972

12. Feldman O, Meir M, Shavit D, Idelman R, Shavit I. Exposure to a surrogate measure of contamination from simulated patients by emergency department personnel wearing personal protective equipment. JAMA. 2020;323(20):2091. doi:10.1001/jama.2020.6633

13. Napoli PE, Nioi M, d'Aloja E, Fossarello M. The ocular surface and the coronavirus disease 2019: does a dual 'ocular route' exist? J Clin Med. 2020;9(5):1269. doi:10.3390/jcm9051269

14. Leung NH, Chu DK, Shiu EY, et al. Respiratory virus shedding in exhaled breath and ecacy of face masks. Nat Med. 2020.

15. Asadi S, Bouvier N, Wexler AS, Ristenpart WD. The coronavirus pandemic and aerosols: does COVID-19 transmit via expiratory particles? Aerosol Sci Technol. 2020;1-4. doi:10.1080/ 02786826.2020 .1749229

16. Guzman M Bioaerosol size effect in COVID-19 transmission. Preprints; 2020:2020040093.

17. World Health Organization. Rational use of personal protective equipment for Coronavirus Disease (COVID-19) and considerations during severe shortages: interim guidance, 6 April 2020; No. WHO/ 2019-nCov/IPC_PPE_use/2020.3. Geneva, Switzerland: World Health Organization; 2020.

18. Chen X, Shang Y, Yao S, Liu R, Liu H. Perioperative care provider's considerations in managing patients with the COVID-19 infections. Transl Perioper Pain Med. 2020;15:216-224. 


\section{Publish your work in this journal}

Clinical Ophthalmology is an international, peer-reviewed journal covering all subspecialties within ophthalmology. Key topics include: Optometry; Visual science; Pharmacology and drug therapy in eye diseases; Basic Sciences; Primary and Secondary eye care; Patient Safety and Quality of Care Improvements. This journal is indexed on PubMed

Submit your manuscript here: https://www.dovepress.com/clinical-ophthalmology-journal
Central and CAS, and is the official journal of The Society of Clinical Ophthalmology (SCO). The manuscript management system is completely online and includes a very quick and fair peer-review system, which is all easy to use. Visit http://www.dovepress.com/ testimonials.php to read real quotes from published authors. 